\title{
Electrode Surface Potential-Driven Protein Adsorption and Desorption through Modulation of Electrostatic, van der Waals, and Hydration Interactions
}

\author{
P.A. Fritz,* B. Bera, J. van den Berg, I. Visser, J.M. Kleijn, R.M. Boom, and C.G.P.H. Schroën
}

Cite This: Langmuir 2021, 37, 6549-6555

Read Online

ACCESS | Llll Metrics \& More | 国 Article Recommendations | sl Supporting Information

ABSTRACT: When proteins in aqueous solutions are exposed to solid substrates, they adsorb due to the dynamic interplay of electrostatic, van der Waals, and hydration interactions and do so in a rather irreversible fashion, which makes protein recovery troublesome. Here, we use a gold electrode as the solid substrate and modulate the surface potential to systematically induce protein adsorption as well as partial desorption. We use different methods such as surface plasmon resonance, atomic force microscopy, and electrowetting and show that biasing the electrode to more negative potentials (by $-0.4 \mathrm{~V}$ compared to the open-circuit potential at $\mathrm{pH} 6$ ) results

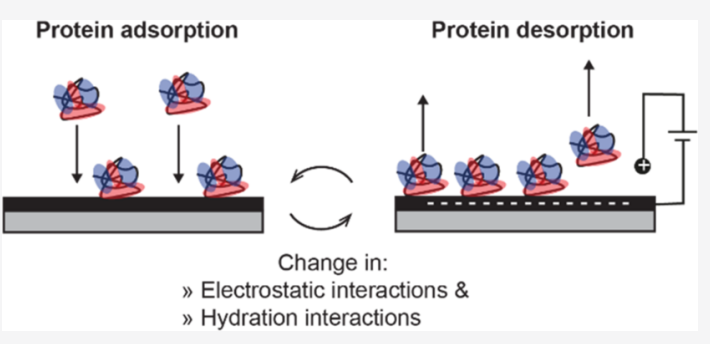
in an increased adsorption barrier of $6 \mathrm{~kJ} \mathrm{~mol}^{-1}$ for the negatively charged protein $\beta$-lactoglobulin. Further, we clearly demonstrate that this is due to an increased double layer potential of $-0.06 \mathrm{~V}$ and an increase in hydration repulsion. This indicates that an electric potential can directly influence surface interactions and thus induce partial $\beta$-lactoglobulin desorption. These observations can be the basis for biosensors as well as separation technologies that use only one trigger to steer protein ad- and desorption, which is low in energy requirement and does not generate large waste streams, as is the case for standard protein separation technologies.

\section{INTRODUCTION}

Although protein adsorption to and desorption from solid interfaces are of great importance in many application fields such as biotechnology, biophysics, medicine, pharmaceutics, and food science, the underlying mechanisms are far from being resolved due to the complexity of the interactions involved. The system in question consists of multiple components (e.g., surface, protein, and solvent) having different properties (e.g., polarity and charge), which, in turn, depending on the environment they are exposed to $(\mathrm{pH}$, isoelectric strength, and temperature), contribute in various ways to system-specific interactions. Furthermore, these effects occur dynamically: when a solid surface is brought into contact with a protein solution, the surface will first be wetted by solvent molecules. Subsequently, proteins diffuse into the nearsurface fluid, replace solvent molecules, and adsorb, leading to an adsorbed layer where conformational rearrangements and replacements can still occur. ${ }^{1-3}$

In addition to the general nature of proteins discussed above, they can form multiple contact points with the surface, leading to a Gibbs free energy of adsorption in the range of tens of $R T$ per mole of protein, with $R$ being the universal gas constant and $T$ the temperature., ${ }^{3,4}$ For example, for $\beta$ lactoglobulin, values between $-5 \mathrm{~kJ} / \mathrm{mol}(-2 R T)$ and $-55 \mathrm{~kJ} /$ mol $(-22 R T)$ depending on the surface ${ }^{5-7}$ have been reported, where the negative sign denotes an attractive interaction between proteins and the surface. ${ }^{3,4}$ Key factors in defining the origin and strength of the binding of proteins to surfaces are electrostatic interactions as well as van der Waals attraction and hydration interaction. ${ }^{8-11}$ Once proteins adsorb, desorption is challenging due to structural rearrangements and relaxation at the interface over time, ${ }^{3}$ which also influences the interaction strength. For example, the maximum desorption for $\beta$-lactoglobulin upon being rinsed with a buffer could be achieved after around $1500 \mathrm{~s}$ after adsorption at a concentration of $5 \times 10^{-8} \mathrm{M}$. This point, where initially irreversibly adsorbed proteins rearrange into reversible species due to crowding, is defined as the critical surface coverage. For shorter as well as longer adsorption times, the amount of protein that could be removed is reported to be less. ${ }^{12}$

Although elution with a buffer is widely used to remove proteins from a surface, it is not effective in many cases because of the high adsorption energy that has to be overcome by using high salt concentrations or extreme $\mathrm{pH}$ (both low and high), leading to the necessity of post-treatments. Alternatively, it has been shown that electrochemical stimuli can directly affect the solid surface potential and thus influence ad- and desorption of molecules including ions and thiols. On a gold electrode, the double layer potential could, for example, be

Received: March 24, 2021

Revised: April 22, 2021

Published: May 19, 2021 

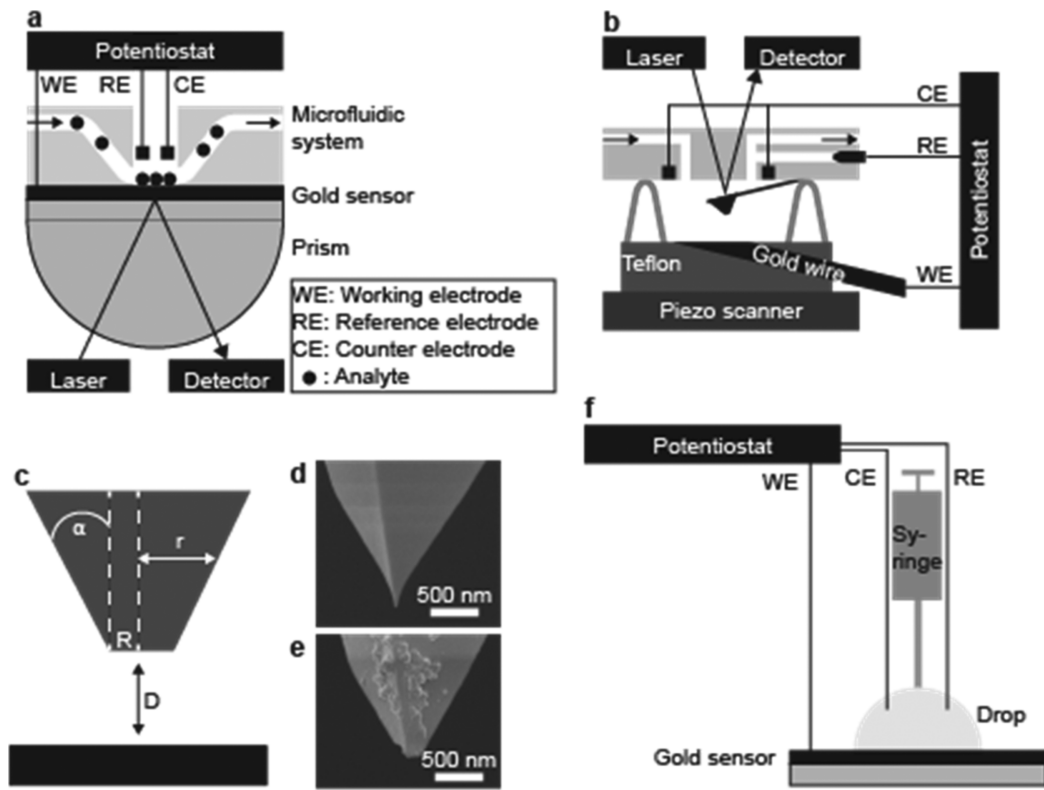

Figure 1. Overview of experimental setups and details of probes. (a) Electrochemical SPR. (b) Electrochemical atomic force spectroscopy. (c) Truncated cone-shaped probe used for atomic force spectroscopy with angle $\alpha$ and radius $R_{\mathrm{p}}$ at the plane facing the gold substrate. (d,e) SEM images of the pointed and blunted atomic force spectroscopy probe, respectively. (f) Electrowetting setup.

shifted between -30 and $20 \mathrm{mV}$ when varying the applied potential from -0.3 to $0.5 \mathrm{~V}$ versus a $\mathrm{Ag} / \mathrm{AgCl}(3 \mathrm{M})$ reference electrode. ${ }^{13-17}$ Further, thiols could be either chemior physisorbed depending on the applied potential. ${ }^{18,19}$

Here, we show that the electrically induced change in surface interactions can be used to manipulate protein ad- and desorption. While the electrochemical manipulation of protein adsorption has been reported to some extent, ${ }^{10,20,21}$ desorption has never been reported for gold surfaces that do not contain electrochemically responsive coatings. ${ }^{22,23}$ Our approach uses a unique combination of atomic force microscopy (AFM), electrowetting, ${ }^{24}$ and surface plasmon resonance (SPR). This allows us to systematically investigate and quantify the fundamental effect of the surface potential on the interactions driving protein ad- and partial desorption. We find that both electrostatic and hydration interactions change based on the applied potential and that as a result, the protein adsorption barrier can be changed by $6 \mathrm{~kJ} \mathrm{~mol}^{-1}$. These findings can be directly applied to improve biosensors and separation technologies $^{25}$ (e.g., electrochemical separation ${ }^{26,27}$ and electrochemically modulated liquid chromatography ${ }^{28-32}$ ).

\section{MATERIALS AND METHODS}

$\boldsymbol{\beta}$-Lactoglobulin Purification and Characterization. $\beta$-Lactoglobulin $\left(M_{\mathrm{w}} 18.4 \mathrm{kDa}\right)$ was purified from whey protein containing $72.4 \%$ protein following Mailliart and Ribadeau-Dumas. ${ }^{33}$ The whey protein solution was adjusted to $\mathrm{pH} 2$ and a $14 \%$ sodium chloride concentration; after overnight stirring, the solution was diluted 2-fold and centrifuged for $10 \mathrm{~min}$ at $10^{4} \mathrm{~g}$. The sodium chloride concentration of the supernatant was subsequently adjusted to $23 \%$ and again centrifuged. The pellet was resuspended, the $\mathrm{pH}$ was adjusted to 7 , and the resulting $\beta$-lactoglobulin solution was dialyzed against water and freeze-dried for storage. The purity of $\beta$ lactoglobulin in the final product was measured using highperformance liquid chromatography (HPLC) and was at least 99.9\% (Figure S1a). The isoelectric point was 4.6 (Zetasizer measurements in the $\mathrm{pH}$ range 3-8; Figure $\mathrm{S1b}$ ).

Surface Plasmon Resonance. An SPR device from Kinetic Evaluation Instruments (The Netherlands) was used in combination with an electrochemical cell (Figure 1a). First, the baseline was measured while cycling $5 \mathrm{mM}$ sodium chloride solution at $\mathrm{pH} 6$ through the cuvette, and the SPR signal was recorded. Subsequently, a $10 \mathrm{mg} / \mathrm{L} \beta$-lactoglobulin solution with $5 \mathrm{mM}$ sodium chloride ( $\mathrm{pH} 6$ ) was introduced into the cuvette. As a reference measurement, the same experiment was conducted without protein; these values were subtracted from those obtained for the $\beta$-lactoglobulin solution to identify the signal related to protein only. The SPR signal was converted by dividing it by 122 to obtain the amount of surfacebound protein in $\mathrm{mg} \mathrm{m}^{-2}$.

For electrochemical measurements, the gold surface of the SPR chip (SSENS, The Netherlands) functioned as the working electrode, a platinum wire was the reference electrode, and a stainless-steel plate was the counter electrode. The reference electrode was calibrated using a $\mathrm{Ag} / \mathrm{AgCl}(3 \mathrm{M})$ reference electrode at $0.3 \mathrm{~V}$ versus $\mathrm{Ag} / \mathrm{AgCl}$ at $\mathrm{pH}$ 6. The open-circuit potential (OCP) of the gold electrode at $\mathrm{pH} 6$ and $5 \mathrm{mM} \mathrm{NaCl}$ was $0.1 \mathrm{~V}$ versus $\mathrm{Ag} / \mathrm{AgCl}(3 \mathrm{M})$.

Adsorption (typically $3 \mathrm{~h}$ ) was measured with respect to different applied potentials to the gold electrode or for alternating potentials. Typically, the OCP would then be applied for 10 to $1800 \mathrm{~s}$, followed by $-0.4 \mathrm{~V}$ for $1800 \mathrm{~s}$, which was repeated four times. All experiments were conducted in independent triplicates. The increase in the energy barrier for adsorption $(\Delta E)$ upon applying $-0.4 \mathrm{~V}$ was determined using eq $1^{3,34}$

$$
\frac{j_{-0.4 \mathrm{~V}}}{j_{\mathrm{OCP}}}=\mathrm{e}^{-\Delta E / R T}
$$

where $j_{-0.4 \mathrm{~V}}$ and $j_{\mathrm{OCP}}$ represent the initial protein adsorption rate at an applied potential of $-0.4 \mathrm{~V}$ versus OCP and at the OCP, respectively. The initial protein adsorption rate was obtained from a linear fit of the initial increase in the surface concentration.

Atomic Force Spectroscopy. A Bruker Nanoscope Multimode scanning probe microscope was used in combination with an electrochemical fluid cell (Figure $1 \mathrm{~b}$ ). To obtain a truncated conical blunted tip, a pointed silicon AFM probe (MikroMasch, NSC36) was scratched on a silicon oxide surface for $1 \mathrm{~h}$ using the AFM probe in the contact mode at a deflection setpoint of $8 \mathrm{~V}$ (Figure $1 \mathrm{c}-\mathrm{e}$ ). ${ }^{35}$ After scratching, the probe radius $R_{\mathrm{p}}$ was $70 \mathrm{~nm}$. The cantilever spring constant was found to be around $0.6 \mathrm{~N} \mathrm{~m}^{-1}$ before and after each measurement. 
Gold electrodes were obtained by fixing a gold wire in a Teflon sample holder and polishing the cross-section $\left(2 \mathrm{~mm}^{2}\right)$ using a $40 \mathrm{~nm}$ silica suspension in water (OP-U suspension, Struers bv, Denmark). This resulted in relatively flat gold surfaces containing areas with a peak-to-valley distance of less than $5 \mathrm{~nm}$ over an area of $0.1 \mu \mathrm{m}^{2}$, as determined by AFM imaging in the contact mode with a standard nitride tip. ${ }^{17}$ The cross-section of the gold wire was used as the working electrode, while a platinum wire was used as the counter electrode and a $3 \mathrm{M} \mathrm{Ag} / \mathrm{AgCl}$ electrode as the reference electrode (Figure $1 \mathrm{~b}$ ). As the electrolyte, a $5 \mathrm{mM}$ sodium chloride solution at $\mathrm{pH} 6$ was used after degassing by sonication for $30 \mathrm{~min}$. With a syringe, $10 \mathrm{~mL}$ of the solution was flushed through the AFM cell. Subsequently, the cell was closed, and the system was left to equilibrate for $10 \mathrm{~min}$. Thereafter, force curves were measured over $500 \mathrm{~nm}$ at a ramp rate of $1 \mathrm{~Hz}$, biasing the working electrode to 0 , $-0.1,-0.2,-0.3$, and $-0.4 \mathrm{~V}$ versus OCP in turn. The data were converted into the normalized force $(F / R)$ by using the probe radius.

Electrowetting. The contact angles were measured using a drop tensiometer (Teclis, France) and the same gold substrates as those used in the SPR experiments. Also, here, the gold substrates were used as the working electrodes, while as counter and reference electrodes, $\mathrm{Pt}$ wires were immersed into the drop $(\mathrm{NaCl} 5 \mathrm{mM})$, as indicated in Figure 1f. The drop was regulated using a $16 \mathrm{G}$ needle (Teclis, France) at $1 \mathrm{~mm}^{3} \mathrm{~s}^{-1}$, and advancing and receding contact angles on the left and right of the side-view image of the drop were measured. After a period of $50 \mathrm{~s}$, a potential ramp from 0 to $-0.5 \mathrm{~V}$ versus $\mathrm{Ag} /$ $\mathrm{AgCl}(3 \mathrm{M})$ was applied to the working electrode using $0.1 \mathrm{~V}$ intermediate steps.

All measurements have been performed at room temperature (25 $\left.{ }^{\circ} \mathrm{C}\right)$.

\section{RESULTS AND DISCUSSION}

A typical $\beta$-lactoglobulin adsorption curve measured using SPR ( Figure 1a) is depicted in Figure 2a. Initially, the negatively charged (Figure S1b) $\beta$-lactoglobulin adsorbs quickly (after 60 $\mathrm{s}$ of baseline measurement); after around $3 \mathrm{~min}$, the surface becomes saturated, and the adsorption rate decreases (Figure $2 \mathrm{a}$ inset). The maximum adsorbed amount when no potential (NP) or the OCP is applied is comparable with values previously found using ellipsometry $\left(2.7 \mathrm{mg} \mathrm{m}^{-2}\right){ }^{12,36,37}$ Under a potential of $-0.4 \mathrm{~V}$, proteins adsorb at a much slower rate, indicating an increase in the adsorption barrier by $6.3 \mathrm{~kJ}$ $\mathrm{mol}^{-1}$ with respect to OCP conditions. Furthermore, the maximum adsorbed amount after $3 \mathrm{~h}$ is reduced to $1.0 \mathrm{mg} \mathrm{m}^{-2}$ (Figure 2a).

Activating the potential from open circuit conditions to -0.4 $\mathrm{V}$ after adsorption for $1800 \mathrm{~s}$ induces the partial desorption of $\beta$-lactoglobulin (Figure 2d); the amount of surface-bound proteins decreases by up to $15 \%$, which becomes slightly less upon repeated operations (Figure $2 \mathrm{e}$ ). The actual amount that can be desorbed depends on the adsorption time, with a maximum at $1800 \mathrm{~s}$ (Figure 2f). This observation can be related to an interplay of the available surface area for proteinsurface interactions and residence time. Three different phases can be distinguished as proposed by Rabe et al. ${ }^{12}$ (i) At a low surface coverage, $\beta$-lactoglobulin adsorbs irreversibly (Figure 2b) since the large amount of the free surface allows for multiple contact points and spreading. (ii) Beyond a critical surface coverage, leading to crowding, the number of contact points per protein decreases, which increases the desorption rate (Figure 2c). (iii) Conformational rearrangements (and possibly intra-protein interactions) of the molecules in the adsorption layer occurring in the second phase eventually result in relaxation and a decreased desorption rate constant. The increase in desorption during the second phase was also observed when eluting with a buffer. ${ }^{12}$ At a higher inflow a
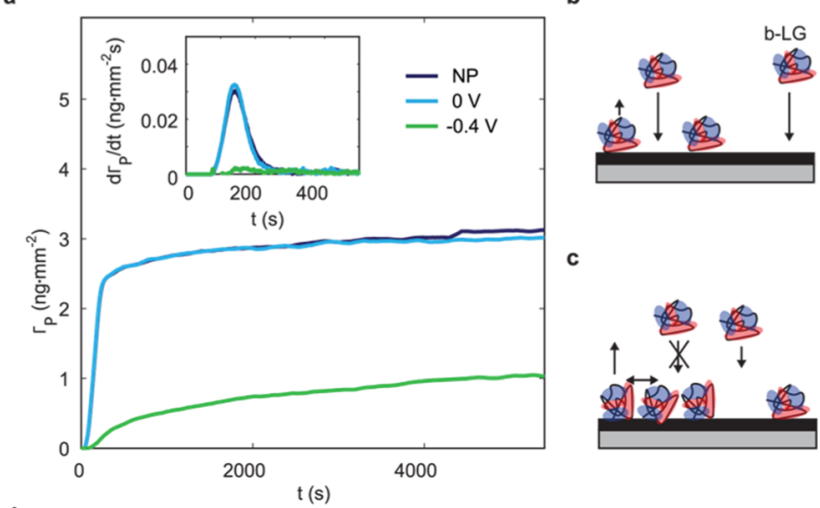

c
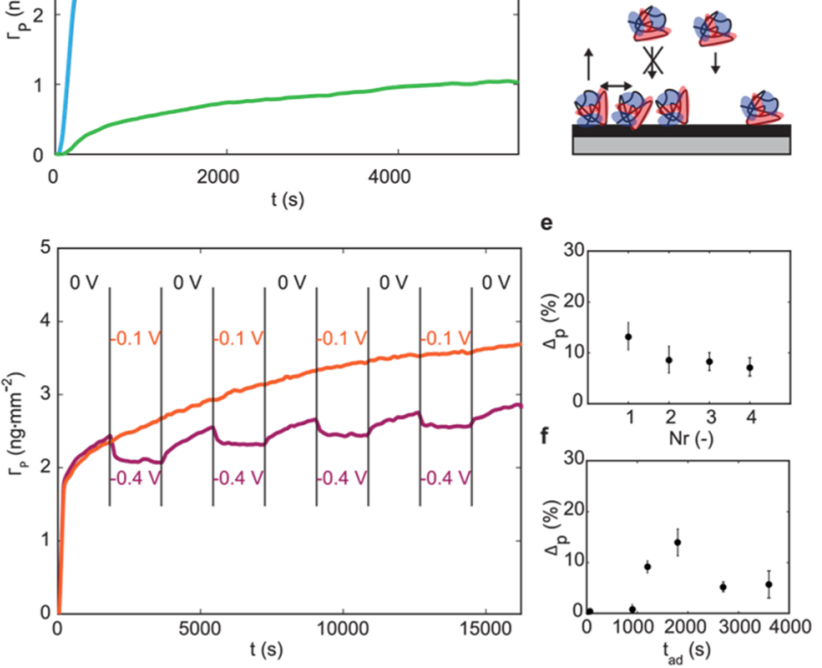

Figure 2. Electrochemically modulated protein adsorption and desorption. (a) Adsorbed amount of protein and adsorption rate (inset) over time at different applied potentials leading to an extra adsorption barrier of $6.3 \mathrm{~kJ} \mathrm{~mol}^{-1}$ at $-0.4 \mathrm{~V}$ relative to the OCP (NP is no potential applied). (b) Protein adsorption to a surface with low coverage. (c) Protein adsorption to a surface with high coverage. (d) $\beta$-Lactoglobulin adsorption at $0 \mathrm{~V}$ and desorption at $-0.1 \mathrm{~V}$ (orange) and $-0.4 \mathrm{~V} v$ S OCP (purple). (e) Desorption efficiency after repeated adsorption for $1800 \mathrm{~s}$ at $0 \mathrm{~V} v \mathrm{O}$ OCP and subsequent desorption at $-0.4 \mathrm{~V} v s$ OCP. (f) Desorption efficiency of the first cycle when -0.4 $\mathrm{V} v s \mathrm{OCP}$ is applied depending on different adsorption times.

concentration of protein, $\beta$-lactoglobulin adsorption increases, for example, to $3.5 \mathrm{mg} \mathrm{m}^{-2}$ for an inflow concentration of 40 $\mathrm{mg} / \mathrm{L}$ and an adsorption time of $1800 \mathrm{~s}$. The desorption efficiency slightly increases as well with the increasing protein concentration, indicating an increase in loosely bound protein at the surface, likely due to additional crowding (Figure S2). An increase of protein adsorption beyond the monolayer coverage can be related to surface roughness. ${ }^{38}$

The mechanisms related to the change in adsorption and desorption behaviors are governed by electrostatic, van der Waals, and hydration interactions, and in the following, we will elaborate how they are impacted by the applied potential. Furthermore, we will discuss aspects to be considered when aiming at increasing the desorption efficiency.

The repulsive forces, measured with AFM (Figure 1b), between a negatively charged silicon probe and the gold electrode increase upon applying a more negative potential to the latter (Figure $3 \mathrm{a}$ ). If we switch the potential from 0 to -0.4 $\mathrm{V}$, repulsion increases from 0.2 to $9 \mathrm{mN} \mathrm{m}^{-1}$ at $3 \mathrm{~nm}$ from the surface, and the distance over which the repulsive forces decay to practically zero (cutoff value: $0.01 \mathrm{mN} \mathrm{m}^{-1}$ ) increases from around 5 to $26 \mathrm{~nm}$.

In order to explain the energy distance curves measured with AFM, we consider the total interaction potential to be a combination of electrostatic, van der Waals, and hydration 


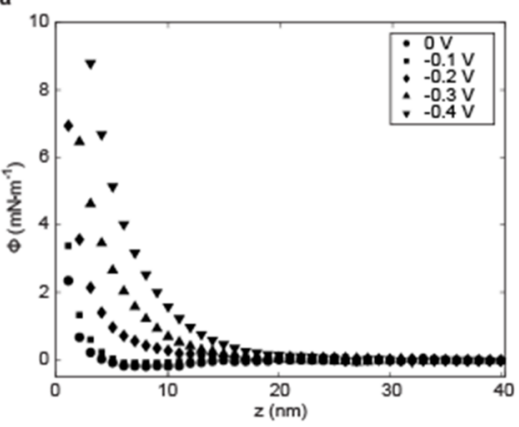

b

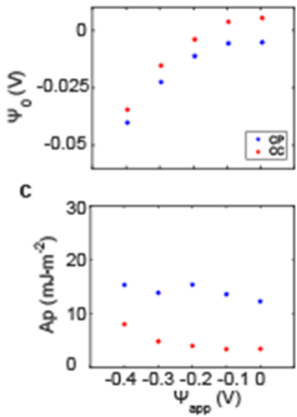

Figure 3. Surface interactions between the gold electrode and silicon probe. (a) Measured energy-distance curves between the truncated silicon probe and gold electrode biased at potentials ranging from 0 to $-0.4 \mathrm{~V} v s$ OCP. The standard deviation ranges are between 0.5 and 2 $\mathrm{mN} \mathrm{m}^{-1}$ (Figure S5). (b) Double layer potential $\psi_{0}$ and (c) hydration repulsion amplitude $A_{\mathrm{p}}$ obtained after fitting interaction energy curves (Figure S5) either using constant potential (CP, blue) or constant charge $(\mathrm{CC}, \mathrm{red})$ boundary conditions.

interactions between two negatively charged surfaces. The change in electrostatic potential $\psi$ in the diffuse double layer in the perpendicular direction $(z)$ from each surface follows from the nonlinear Poisson-Boltzmann equation ${ }^{9}$ (eq 2) for an $m$ / $m$ electrolyte, such as $\mathrm{NaCl}$.

$$
\frac{\mathrm{d}^{2} \psi}{\mathrm{d} z^{2}}=\kappa^{2} \frac{k T}{e} \sinh \left(\frac{e}{k T} \psi\right)
$$

where $\kappa^{-1}$ is the Debye screening length, $e$ is the elementary charge, $\varepsilon$ is the relative dielectric constant ( 78.54 for water at $\left.25{ }^{\circ} \mathrm{C}\right), \varepsilon_{0}$ is the permittivity of vacuum, and $k$ is the Boltzmann constant. In Figure $4 \mathrm{a}$, potential profiles for different boundary conditions are depicted. It becomes evident that with a more negative surface potential, the absolute potential drop and the distance at which the potential drops to zero become larger. In Figure $4 b$, the relation between the ion distribution and the potential drop is sketched, and the three distinct regions ranging from the Stern layer (A) that is in close proximity to the surface to the diffuse layer (B) and the bulk (C) region are indicated. At large distances $D$ (in the $z$ direction) between the two negatively charged surfaces, the potential drop is fully developed, and no interaction is detected, but with increasing proximity, the excess of ions near the surfaces leads to repulsion. ${ }^{39}$ This can be calculated using eqs 3 and 4,,35 (results shown in Figure 4c,d for constant potential and constant charge boundary conditions, respectively).

$$
\begin{aligned}
& \Pi_{\mathrm{El}}(\mathrm{D})=2 n_{0} k T\left[\cosh \left(\frac{e \psi}{k T}\right)-1\right]-\frac{\varepsilon \varepsilon_{0}}{2}\left(\frac{\mathrm{d} \psi}{\mathrm{d} z}\right)^{2} \\
& \Phi_{\mathrm{El}}=\Pi_{\mathrm{El}}(D) \pi R_{\mathrm{p}}+2 \pi \int_{R}^{\infty} \Pi_{\mathrm{El}}\left(D+\frac{r-R}{\tan \alpha}\right) \mathrm{d} r
\end{aligned}
$$

Using eq 3 , we can calculate the electrostatic part of the disjoining pressure $\left(\Pi_{\mathrm{El}}\right)$ between two flat surfaces, with $n_{0}$ being the number of ions in the bulk $\left(\mathrm{m}^{-3}\right)$ and $D$ the distance between the two plates in the $z$ direction. For calculation of the electrostatic interaction energy $\left(\Phi_{\mathrm{El}}\right)$ between the adsorbing surface and the probe (eq 4), the shape of the probe has been taken into account. As indicated in Figure $1 c, R$ is the radius of the truncated base at the front of the tip, $r$ is the radius at a
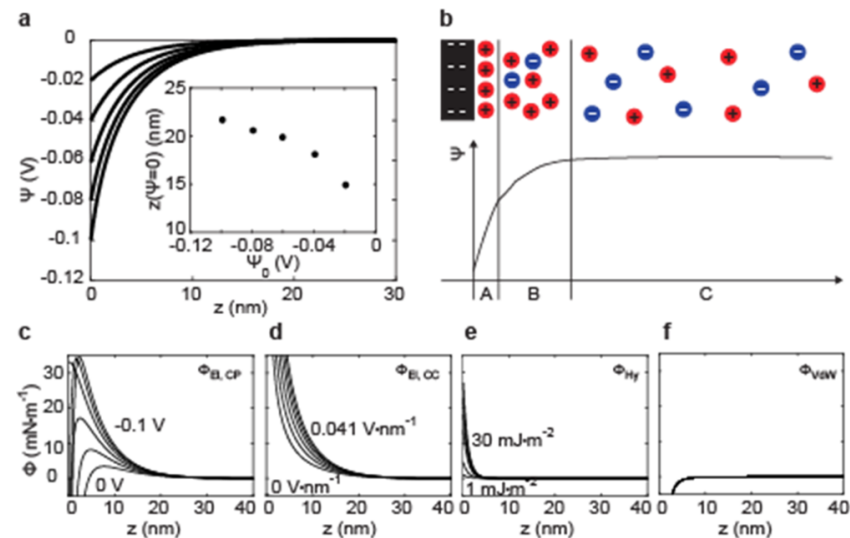

Figure 4. (a) Double layer potential ( $\psi$ ) profile as described by the Poisson-Boltzmann equation with varying surface potentials $\left(\psi_{0}\right.$ between -0.1 and $-0.02 \mathrm{~V})$. Inset: Position at which the potential drops to $0 \mathrm{~V}$ (cutoff: $-5 \times 10^{-4} \mathrm{~V}$ ). (b) Schematic of the ion distribution at the electrode interface within the electric double layer and related potential profile. (c) Electrostatic interaction energy determined for constant potential boundary conditions; the gold electrode was held at $0,-0.2,-0.4,-0.6,-0.8$, or $-1.0 \mathrm{~V}$, and the silica probe was set at $-0.06 \mathrm{~V}$. (d) Constant charge boundary conditions were determined using Graham's equation for values between 0 and $0.041 \mathrm{~V} \mathrm{~nm}^{-1}$ for the gold electrode and $-0.0173 \mathrm{~V}$ $\mathrm{nm}^{-1}$ for the silica surface. (e) Hydration repulsion for a hydration repulsion amplitude between 1 and $30 \mathrm{~mJ} \mathrm{~m}^{-2}$ with a decay length of $1.3 \mathrm{~nm}$. (f) Van der Waals attraction for a Hamaker constant of $5.7 \times$ $10^{-20} \mathrm{~J}$.

random location between the two bases, and $\alpha$ is the cone angle. Since the silicon probe has an oxidized surface layer, we used $-0.06 \mathrm{~V}$ for the surface potential of silica ${ }^{35}$ and used the surface potential or the surface charge of the gold electrode as a fit parameter. At constant potential boundary conditions, a maximum can be observed for the electrostatic interaction. This leads to attractive forces at distances below $8 \mathrm{~nm}$ (Figure $4 \mathrm{c}$ ); this is due to the charge reversal at the boundary (Figures S3 and S4). ${ }^{16}$

Next, we calculate the contribution of the van der Waals interactions to the total interaction energy. The corresponding disjoining pressure and the interaction energy are given by eqs 5 and $6^{9,35}$

$$
\begin{aligned}
& \Pi_{\mathrm{VdW}}(D)=-\frac{A_{\mathrm{H}}}{6 \pi D^{3}} \\
& \Phi_{\mathrm{VdW}}=\Pi_{\mathrm{VdW}}(D) \pi R_{\mathrm{p}}+2 \pi \int_{R}^{\infty} \Pi_{\mathrm{VdW}}\left(D+\frac{r-R}{\tan \alpha}\right) \mathrm{d} r
\end{aligned}
$$

where $A_{\mathrm{H}}$ is the Hamaker constant for this specific combination of phases. The gold-water-silica system has a rather high Hamaker constant $\left(5.7 \times 10^{-20} \mathrm{~J}^{17}\right)$, which leads to strong attraction at distances shorter than $8 \mathrm{~nm}$ (see Figure $4 \mathrm{e})$. This attraction is, however, rarely detected in energydistance curves ${ }^{40}$ as measured here (Figure 3a). This effect has been previously related to either the presence of adlayers or surface roughness, and an inward shift of the onset of the van der Waals interactions in the order of the surface roughness has been proposed. ${ }^{41}$ Furthermore, strong hydration forces are acting in close proximity to the surface. The hydration contribution is known to be more difficult to quantify; 


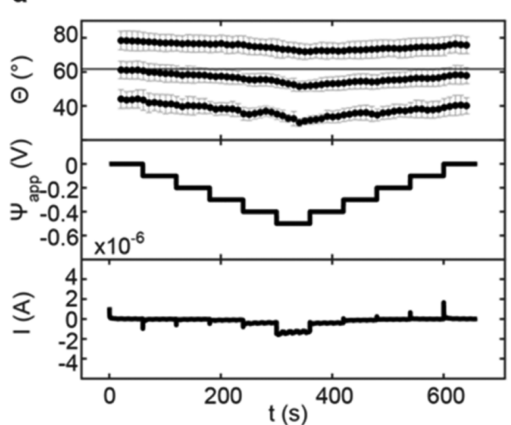

b

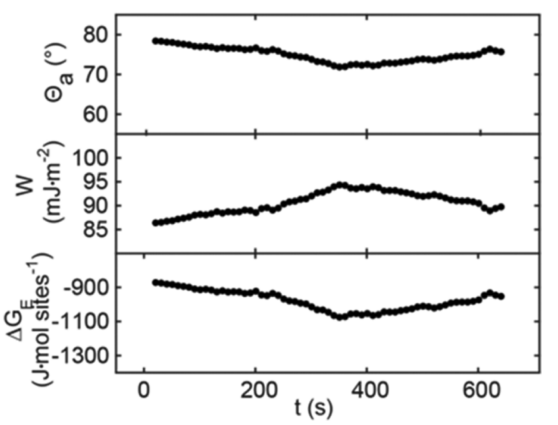

Figure 5. Contact angle of water on a gold electrode as a function of applied potential. (a) Advancing, average, and receding contact angles (top) depending on the applied potential (middle) and current response (bottom). (b) Advancing contact angle (top), resulting work of water adhesion (middle), and free energy of wetting per mole of surface sites (bottom).

hydration contributions of the interaction potential follow eq $7^{42}$

$$
\Phi_{\mathrm{Hy}}=A_{\mathrm{p}} \mathrm{e}^{-D / \lambda_{\mathrm{Hy}}}
$$

where $A_{\mathrm{p}}$ is the amplitude of hydration repulsion, while $\lambda_{\mathrm{Hy}}$ is the decay length. Results for different $A_{\mathrm{p}}$ values at a $\lambda_{\mathrm{Hy}}$ of 1.3 $\mathrm{nm}$ are shown in Figure $4 \mathrm{f}$.

The abovementioned contributions are summed to give the total interaction energy as indicated in eq 8.

$$
\Phi=\Phi_{\mathrm{El}}+\Phi_{\mathrm{VdW}}+\Phi_{\mathrm{Hy}}
$$

The latter has been fitted to the measured energy-distance curves (Figure 3a). For a good fit, we shifted the data points by $3.5 \mathrm{~nm}$ to the right (Figure S5). This can be justified by the surface roughness of both the polished polycrystalline gold electrode and the blunted AFM probe, affecting the precise onset of the interactions. ${ }^{17}$ In addition, factors such as the angle of the flattened cone surface with respect to the gold substrate and the exact value of its radius add to the fitting uncertainties. As suggested by Valtiner et al., ${ }^{41}$ together with the data points, we shifted the onset of the hydration forces, while van der Waals forces are moved by $3.5 \mathrm{~nm}$ to more negative values, resulting in good fits with $R^{2}$ values higher than 0.92 (Figure S5). Nevertheless, a clear dependency of the electric double layer potential (Figures $3 b$ and S4) as well as the hydration repulsion amplitude (Figure $3 c$ ) on the applied potential can be detected. The double layer potential (Figures $3 \mathrm{~b}$ and S4) is more negative with the increasing negative applied potential (leading to more electrostatic repulsion). When applying $-0.4 \mathrm{~V}$, a double layer potential of $-0.04 \mathrm{~V}$ could be achieved. This value is similar compared to the values obtained by Barten et al. ${ }^{17}$ using a colloidal probe in a $1 \mathrm{mM}$ $\mathrm{KNO}_{3}$ electrolyte at $\mathrm{pH} 4.7$ when applying $-0.3 \mathrm{~V}$ versus $\mathrm{Ag} /$ $\mathrm{AgCl}$. Furthermore, the hydration repulsion amplitude increases (Figure 3c), indicating stronger hydration repulsion at more negative potentials. This indicates that not only the ion assembly at the vicinity of the electrode but also the arrangement of water molecules at the surface is influenced, thereby greatly impacting the adsorption and desorption behaviors of proteins also at short distances from the electrode surfaces.

The hydration repulsion effects relate to a change in surface wetting, which has been investigated experimentally at the meso scale through contact angle measurements (Figure 1f). From the advancing contact angle and the surface tension, the work of water adhesion ( $W$, eq 9 ), and the free energy of wetting per mole of surface sites $\left(\Delta G_{E} \text {, eq } 10\right)^{4}$ can be determined.

$$
\begin{aligned}
& W=\gamma_{\mathrm{lv}}\left(1+\cos \theta_{\mathrm{a}}\right) \\
& \Delta G_{E}=\left(\frac{R T}{3}\right) \ln \left[\frac{\left(1-\cos \theta_{\mathrm{a}}\right)^{2}\left(2+\cos \theta_{\mathrm{a}}\right)}{4}\right]
\end{aligned}
$$

where $\gamma_{\mathrm{lv}}$ is the surface tension of water and $\theta_{\mathrm{a}}$ is the advancing contact angle. The advancing contact angle decreases from 80 to $72^{\circ}$ when the applied potential is changed from 0 to $-0.5 \mathrm{~V}$ (Figures 5a and S5) and with it increases the work of water adhesion, and the free energy of wetting becomes more negative (Figure $5 b$ ).

It has been demonstrated that a change in contact angle can be related to a change in the hydrogen bond network between the surface and the water molecules in close proximity to the surface having a strong impact on protein adsorption and desorption. ${ }^{4,43}$ For a gold substrate, it was demonstrated that the majority of water molecules lie flat or perpendicular on the surface, contributing at least one $\mathrm{H}$ atom to the hydrogen bond network. ${ }^{44}$ Hence, it can be concluded that a gold surface has more $\mathrm{H}$ donors than $\mathrm{H}$ acceptors, and it was shown that with an increasing negative potential, this ratio is further distorted. ${ }^{44}$ This could lead to a smaller contact angle and more hydration repulsion under negative applied potentials as demonstrated in this article. Protein adsorption as well as desorption is greatly influenced by hydration interactions; as such, the Berg limit has been identified at a contact angle of $60^{\circ} .{ }^{45,46}$ Below this angle, the surface becomes in general adsorption-resistant due to strong hydration repulsion forces. ${ }^{47}$ Along these lines, hydration interactions have also been identified as a key driving factor for protein desorption acting in close proximity to the surface. ${ }^{11}$ Thus, it is essential when aiming at higher protein desorption efficiencies to improve electrowetting properties of the electrode material. The influence of electrowetting on protein electrode interactions has also been discussed by Barten et al. in relation to the classical Lippman relationship. ${ }^{10}$ Also, electro-responsive coatings can support this aspect, such as poly(ferrocenylsilane) films bearing undecanesulfonate surface moieties allowing a change in the contact angle from $59^{\circ}$ (the reduced state) to $77^{\circ}$ (the oxidized state). ${ }^{48}$

All these findings together indicate that overlapping electric double layers and the arrangement of water molecules near the surface both contribute to repulsive forces at the interface. Furthermore, we conclude that both force contributions can be influenced by an externally applied potential, increasing the 
repulsive forces at increasingly negative applied potential values. This, in turn, influences the adsorption free energy and thus not only decreases protein adsorption but also induces partial protein desorption, especially when close to the critical surface coverage.

\section{CONCLUSIONS}

We present a unique combination of techniques to study the influence of the surface potential of a solid substrate on protein adsorption as well as partial desorption. Upon changing the surface potential with a negative electric bias of $-0.4 \mathrm{~V}$ with respect to the OCP, the adsorption barrier increased by $6.3 \mathrm{~kJ}$ $\mathrm{mol}^{-1}$ due to an increase in electrostatic and hydration repulsion, leading to reduced and slower protein adsorption. Furthermore, the increase in the double layer potential and work of water adhesion also triggered partial protein desorption. These are crucial findings that help elucidate the complex mechanisms behind protein ad- and desorption and how both can be modulated by the surface potential and thus applied in the design of innovative biosensors as well as protein separation technologies that can be loaded and regenerated by the "switch of a button".

\section{ASSOCIATED CONTENT}

\section{s) Supporting Information}

The Supporting Information is available free of charge at https://pubs.acs.org/doi/10.1021/acs.langmuir.1c00828.

HPLC retention peaks of whey protein isolate and purified $\beta$-lactoglobulin, zeta potential measurements of $\beta$-lactoglobulin, protein desorption efficiencies at different protein concentrations measured using SPR, boundary conditions and midplane values at changing distances of two charged objects, constant charge and constant potential fits to AFM force curves, dynamic contact angle data, and droplet diameter change (PDF)

\section{AUTHOR INFORMATION}

\section{Corresponding Author}

P.A. Fritz - Laboratory of Food Process Engineering, Wageningen University, Wageningen 6708 WG, The Netherlands; School of Chemical and Biomedical Engineering, Nanyang Technological University, 637459, Singapore; 이이이.org/0000-0002-4237-9341; Email: pina.fritz@wur.nl

\section{Authors}

B. Bera - Laboratory of Food Process Engineering, Wageningen University, Wageningen 6708 WG, The Netherlands

J. van den Berg - Laboratory of Food Process Engineering, Wageningen University, Wageningen 6708 WG, The Netherlands

I. Visser - Laboratory of Food Process Engineering, Wageningen University, Wageningen 6708 WG, The Netherlands

J.M. Kleijn - Physical Chemistry and Soft Matter, Wageningen University, Wageningen 6708 WE, The Netherlands

R.M. Boom - Laboratory of Food Process Engineering, Wageningen University, Wageningen 6708 WG, The Netherlands
C.G.P.H. Schroën - Laboratory of Food Process Engineering, Wageningen University, Wageningen 6708 WG, The Netherlands

Complete contact information is available at: https://pubs.acs.org/10.1021/acs.langmuir.1c00828

\section{Notes}

The authors declare no competing financial interest.

\section{ACKNOWLEDGMENTS}

We would like to acknowledge the Institute for Sustainable Process Technology (ISPT) for funding our project.

\section{REFERENCES}

(1) Rabe, M.; Verdes, D.; Seeger, S. Understanding Protein Adsorption Phenomena at Solid Surfaces. Adv. Colloid Interface Sci. 2011, 162, 87-106.

(2) Nakanaishi, K.; Sakiyama, T.; Imamura, K. On the Adsorption of Proteins on Solid Surfaces, a Common but Very Complicated Phenomenon. J. Biosci. Bioeng. 2001, 91, 233-244.

(3) Norde, W. Adsorption of Proteins at Solid-Liquid Interfaces. Cells Mater 1986, 25, 267-340.

(4) Vogler, E. A. Protein Adsorption in Three Dimensions. Biomaterials 2012, 33, 1201-1237.

(5) Omanovic, S.; Roscoe, S. G. Interfacial Behavior of $\beta$ Lactoglobulin at a Stainless Steel Surface: An Electrochemical Impedance Spectroscopy Study. J. Colloid Interface Sci. 2000, 227, $452-460$.

(6) Fainerman, V. B.; Miller, R.; Ferri, J. K.; Watzke, H.; Leser, M. E.; Michel, M. Reversibility and Irreversibility of Adsorption of Surfactants and Proteins at Liquid Interfaces. Adv. Colloid Interface Sci. 2006, 123-126, 163-171.

(7) Bonomo, R.; Minim, L.; Coimbra, J.; Fontan, R.; Mendesdasilva, L.; Minim, V. Hydrophobic Interaction Adsorption of Whey Proteins: Effect of Temperature and Salt Concentration and Thermodynamic Analysis. J. Chromatogr. B: Anal. Technol. Biomed. Life Sci. 2006, 844, $6-14$.

(8) Lyklema, J. Fundamentals of Interface and Colloid Science; Academic Press, 2000.

(9) Israelachvili, J. N. Intermolecular and Surface Forces; 3rd ed.; Elsevier, 2011.

(10) Kleijn, J. M.; Barten, D.; Cohen Stuart, M. A. Adsorption of Charged Macromolecules at a Gold Electrode. Langmuir 2004, 20, 9703-9713.

(11) McUmber, A. C.; Randolph, T. W.; Schwartz, D. K. Electrostatic Interactions Influence Protein Adsorption (but Not Desorption) at the Silica-Aqueous Interface. J. Phys. Chem. Lett. 2015, 6, 2583-2587.

(12) Rabe, M.; Verdes, D.; Rankl, M.; Artus, G. R. J.; Seeger, S. A Comprehensive Study of Concepts and Phenomena of the Nonspecific Adsorption of B-Lactoglobulin. ChemPhysChem 2007, 8, 862-872.

(13) Butt, H.-J.; Cappella, B.; Kappl, M. Force Measurements with the Atomic Force Microscope: Technique, Interpretation and Applications. Surf. Sci. Rep. 2005, 59, 1-152.

(14) Butt, H.-J. Analyzing Electric Double Layers with the Atomic Force Microscope. Encyclopedia of Electrochemistry; Wiley-VCH Verlag GmbH \& Co. KGaA: Weinheim, Germany, 2007, pp 225248.

(15) Ricci, M.; Spijker, P.; Voïtchovsky, K. Water-Induced Correlation between Single Ions Imaged at the Solid-Liquid Interface. Nat. Commun. 2014, 5, 4400.

(16) Hillier, A. C.; Kim, S.; Bard, A. J. Measurement of DoubleLayer Forces at the Electrode/Electrolyte Interface Using the Atomic Force Microscope: Potential and Anion Dependent Interactions. J. Phys. Chem. 1996, 100, 18808-18817. 
(17) Barten, D.; Kleijn, J. M.; Duval, J.; Leeuwen, H. P. V.; Lyklema, J.; Cohen Stuart, M. A. Double Layer of a Gold Electrode Probed by AFM Force Measurements. Langmuir 2003, 19, 1133-1139.

(18) Muskal, N.; Mandler, D. The Electrochemistry of Thiol SelfAssembled Monolayers (SAMs) on a Hanging Mercury Drop Electrode (HMDE). Curr. Sep. 2000, 19, 49-54.

(19) Riepl, M.; Mirsky, V. M.; Wolfbeis, O. S. Electrical Control of Alkanethiols Self-Assembly on a Gold Surface as an Approach for Preparation of Microelectrode Arrays. Mikrochim. Acta 1999, 131, 29-34.

(20) Barten, D.; Mieke Kleijn, J.; Cohen Stuart, M. A. Adsorption of a Linear Polyelectrolyte on a Gold Electrode. Phys. Chem. Chem. Phys. 2003, 5, 4258.

(21) Moulton, S. E.; Barisci, J. N.; Bath, A.; Stella, R.; Wallace, G. G. Investigation of Protein Adsorption and Electrochemical Behavior at a Gold Electrode. J. Colloid Interface Sci. 2003, 261, 312-319.

(22) Mu, L.; Liu, Y.; Cai, S.; Kong, J. A Smart Surface in a Microfluidic Chip for Controlled Protein Separation. Chem.-Eur J. 2007, 13, 5113-5120.

(23) Su, X.; Hübner, J.; Kauke, M. J.; Dalbosco, L.; Thomas, J.; Gonzalez, C. C.; Zhu, E.; Franzreb, M.; Jamison, T. F.; Hatton, T. A. Redox Interfaces for Electrochemically Controlled Protein-Surface Interactions: Bioseparations and Heterogeneous Enzyme Catalysis. Chem. Mater. 2017, 29, 5702-5712.

(24) Mugele, F.; Baret, J.-C. Electrowetting: From Basics to Applications. J. Phys. Condens. Matter 2005, 17, R705-R774.

(25) Maharjan, P.; Woonton, B. W.; Bennett, L. E.; Smithers, G. W.; DeSilva, K.; Hearn, M. T. W. Novel Chromatographic SeparationThe Potential of Smart Polymers. Innovative Food Sci. Emerging Technol. 2008, 9, 232-242.

(26) Yoon, H.; Lee, J.; Kim, S.; Yoon, J. Review of Concepts and Applications of Electrochemical Ion Separation (EIONS) Process. Sep. Purif. Technol. 2019, 215, 190-207.

(27) Su, X.; Hatton, T. A. Electrosorption at Functional Interfaces: From Molecular-Level Interactions to Electrochemical Cell Design. Phys. Chem. Chem. Phys. 2017, 19, 23570-23584.

(28) Harnisch, J. A.; Porter, M. D. Electrochemically Modulated Liquid Chromatography: An Electrochemical Strategy for Manipulating Chromatographic Retention. Analyst 2001, 126, 1841-1849.

(29) Porter, M. D.; Takano, H. D. CHROMATOGRAPHY: LIQUID | Electrochemically Modulated Liquid Chromatography. Encyclopedia of Separation Science; Elsevier, 2000, pp 636-646.

(30) Yamini, Y.; Seidi, S.; Rezazadeh, M. Electrical Field-Induced Extraction and Separation Techniques: Promising Trends in Analytical Chemistry-A Review. Anal. Chim. Acta 2014, 814, 1-22.

(31) Collins, C. J.; Arrigan, D. W. M. A Review of Recent Advances in Electrochemically Modulated Extraction Methods. Anal. Bioanal. Chem. 2009, 393, 835-845.

(32) Su, X.; Hatton, T. A. Redox-Electrodes for Selective Electrochemical Separations. Adv. Colloid Interface Sci. 2017, 244, $6-20$.

(33) Mailliart, P.; Ribadeau-Dumas, B. Preparation of BLactoglobulin and b-Lactoglobulin-Free Proteins from Whey Retentate by $\mathrm{NaCl}$ Salting Out at Low PH. J. Food Sci. 1988, 53, 743-745.

(34) Norde, W.; Arai, T.; Shirahama, H. Protein Adsorption in Model Systems. Biofouling 1991, 4, 37-51.

(35) Ebeling, D.; van den Ende, D.; Mugele, F. Electrostatic Interaction Forces in Aqueous Salt Solutions of Variable Concentration and Valency. Nanotechnology 2011, 22, 305706.

(36) Marsh, R. J.; Jones, R. A. L.; Sferrazza, M. Adsorption and Displacement of a Globular Protein on Hydrophilic and Hydrophobic Surfaces. Colloids Surf., B 2002, 23, 31-42.

(37) Elofsson, U. M.; Paulsson, M. A.; Arnebrant, T. Adsorption of B-Lactoglobulin A and B in Relation to Self-Association: Effect of Concentration and PH. Langmuir 1997, 13, 1695-1700.

(38) Rechendorff, K.; Hovgaard, M. B.; Foss, M.; Zhdanov, V. P.; Besenbacher, F. Enhancement of Protein Adsorption Induced by Surface Roughness. Langmuir 2006, 22, 10885-10888.
(39) Bard, A. J.; Faulkner, L. R. Electrochemical MethodsFundamentals and Applications, 2nd ed.; John Wiley \& Sons, Inc., 1944.

(40) Giesbers, M.; Kleijn, J. M.; Cohen Stuart, M. A. The Electrical Double Layer on Gold Probed by Electrokinetic and Surface Force Measurements. J. Colloid Interface Sci. 2002, 248, 88-95.

(41) Valtiner, M.; Banquy, X.; Kristiansen, K.; Greene, G. W.; Israelachvili, J. N. The Electrochemical Surface Forces Apparatus: The Effect of Surface Roughness, Electrostatic Surface Potentials, and Anodic Oxide Growth on Interaction Forces, and Friction between Dissimilar Surfaces in Aqueous Solutions. Langmuir 2012, 28, 1308013093.

(42) Kilpatrick, J. I.; Loh, S.-H.; Jarvis, S. P. Directly Probing the Effects of Ions on Hydration Forces at Interfaces. J. Am. Chem. Soc. 2013, 135, 2628-2634.

(43) Besseling, N. A. M. Theory of Hydration Forces between Surfaces. Langmuir 1997, 13, 2113-2122.

(44) Velasco-Velez, J.-J.; Wu, C. H.; Pascal, T. A.; Wan, L. F.; Guo, J.; Prendergast, D.; Salmeron, M. The Structure of Interfacial Water on Gold Electrodes Studied by X-Ray Absorption Spectroscopy. Science (80-. ) 2014, 346, 831-834.

(45) Berg, J. M.; Eriksson, L. G. T.; Claesson, P. M.; Borve, K. G. N. Three-Component Langmuir-Blodgett Films with a Controllable Degree of Polarity. Langmuir 1994, 10, 1225-1234.

(46) Vogler, E. A. Structure and Reactivity of Water at Biomaterial Surfaces. Adv. Colloid Interface Sci. 1998, 74, 69-117.

(47) Schwierz, N.; Horinek, D.; Liese, S.; Pirzer, T.; Balzer, B. N.; Hugel, T.; Netz, R. R. On the Relationship between Peptide Adsorption Resistance and Surface Contact Angle: A Combined Experimental and Simulation Single-Molecule Study. J. Am. Chem. Soc. 2012, 134, 19628-19638.

(48) Dos Ramos, L.; de Beer, S.; Hempenius, M. A.; Vancso, G. J. Redox-Induced Backbiting of Surface-Tethered Alkylsulfonate Amphiphiles: Reversible Switching of Surface Wettability and Adherence. Langmuir 2015, 31, 6343-6350. 